\title{
Emphysematous pyelonephritis with complete duplication of the left urinary tract
}

\author{
Yasuto Yamasaki MD, PhD; ; Shigehiko Koga MD, PhD; 'suzo Minami MD; Hideki SakaiMD, PhD;
}

*Department of Urology, Sasebo Central Hospital, Nagasaki, Japan; †Department of Urology, Japanese Red Cross Nagasaki Genbaku Hospital, Nagasaki, Japan; §Department of Urology, Kuroki Hospital, Nagasaki, Japan; ”Department of Nephro-Urology, Nagasaki University School of Biomedical Sciences, Nagasaki, Japan

Cite as: Can Urol Assoc J 2011;5(6):E101-E103; http://dx.doi.org/10.5489/cuaj.10133

\section{Abstract}

Emphysematous pyelonephritis (EPN) is a life-threatening bacterial infection and should be treated rapidly and carefully. We report a case of EPN with complete duplication of the left urinary tract. A 68-year-old woman was admitted to our hospital complaining of high-grade fever and left flank pain. An abdominal computed tomography scan showed gas was presented in the renal parenchyma, not only the pelvis and ureter. Based on these findings, a diagnosis of left EPN was made. A partial nephrectomy of the affected left upper pole moiety was performed and the patient underwent additional medical management. Other 83 cases of EPN from the Japanese literature were reviewed.

\section{Introduction}

Emphysematous pyelonephritis (EPN) is a severe necrotizing infection caused by gas-forming organisms that lead to the collection of gas within the renal parenchyma, collecting system or perirenal space. In 1898, Kelly and MacCullum reported the first case of EPN, ${ }^{1}$ and demonstrated the presence of gas bubbles and subsequent drainage of an infected kidney using ureteral catheterization. This condition is frequently seen in patients with uncontrolled diabetes mellitus. We present the first case of EPN in a patient with complete duplication of the left urinary tract. We also review the associated literature to evaluate the prognosis of EPN.

\section{Case presentation}

A 68-year-old woman was admitted to our hospital complaining of high-grade fever and left flank pain. There was marked tenderness in the left flank abdomen along with cost vertebral angle. She did not have diabetes mellitus. Laboratory findings on admission were: white blood cells count $3900 / \mathrm{mm}^{3}$ with a shift to the left; C-reactive protein $18.0 \mathrm{mg} / \mathrm{dL}$; urinalysis $>100$ white and red cells per high- power field. Abdominal x-ray showed an abnormal gas pattern in the left kidney area. An abdominal computed tomography (CT) scan showed that gas was present in the left renal parenchyma and loculated gas was present in the pelvis and ureter (Fig. 1). As the result of these findings, a diagnosis was made of left EPN. Fluids and broad-spectrum antibiotics were administered. A percutaneous nephrostomy was performed through the left kidney under ultrasound guidance. Cultures of pus yielded Escherichia coli and Bacteroides spp. Blood culture was negative. Intravenous pyelography and antegrade pyelography from percutaneous drainage showed complete duplication of the left urinary tract and complete obstruction of the upper duplicated tract near the bladder neck (Fig. 2). We examined cystoscopy to identify the ureteric orifice from the upper pole moiety, but this was not possible.

The patient relatively improved after the insertion of a percutaneous drain. On nuclear imaging, we found the patient had a nonfunctioning in the left upper pole moiety and a functioning in the left lower pole moiety. A CT scan revealed the left lower pole moiety was not affected by EPN. A partial nephrectomy of the affected left upper pole moiety was performed of the flank incision. Numerous pus-filled gas bubbles spurted from the perinephric space during the operation. The left renal upper pole moiety was gray. Segmental arterial and venous branches to the diseased portion of the kidney are ligated and divided. It was easy to remove the left upper pole moiety. The ureter to the upper pole moiety was divided as far inferiorly as possible. The histological examination revealed a severe acute inflammatory process, with the formations of abscesses from the renal pelvis to the perinephric fatty tissue. The postoperative course was satisfactory.

\section{Discussion}

Emphysematous pyelonephritis is a rare and life-threatening bacterial infection associated with gas formation in the renal parenchyma; this gas formation can also occur in the perire- 


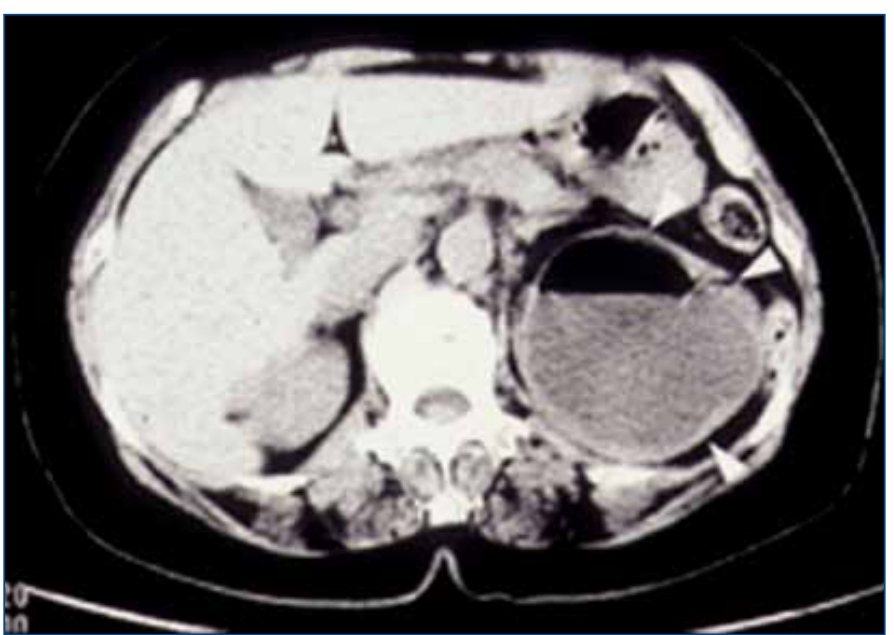

Fig. 1. Computed tomography scan shows the presence of gas collection in left renal parenchyma (arrow).

nal space or collecting system. The pathogenesis of the EPN is still not clear. Nevertheless, three conditions are believed to be important in the development of gases in EPN: (1) the presence of gas-forming bacteria; (2) high level of glucose in tissues; and (3) impaired tissue perfusion. ${ }^{2}$

Diabetics compromise $90 \%$ of patients with EPN, and females outnumber males considerably. ${ }^{3}$ Obstruction of the urinary tract has been reported in $29 \%$ of patients with EPN. ${ }^{4}$ Whether calculus disease is responsible for urinary tract obstruction in these cases remains unclear. ${ }^{5,6}$ Nondiabetic patients with EPN have a higher incidence of urinary obstruction.

Emphysematous pyelonephritis requires a radiological diagnosis, with CT being the most definitive modality. Abdominal x-ray and ultrasound can also be used to diagnosis EPN. The gold standard is an abdominal CT, as it will also show the presence and localization of the gas. It will also show the extent of the destruction of the renal parenchyma. As well as diagnosing and staging EPN, CT is valuable for monitoring the response to treatment. The image might show resolution of the gas and abscesses or the development of new lesions.

Wan and colleagues defined two types of EPN on the basis of radiological findings on CT: ${ }^{7}$ Type 1: renal necrosis with presence of gas but no fluid; and Type 2: parenchymal gas associated with fluid in renal parenchyma, perinephric space or collecting system. Type 1 EPN has a high mortality rate $(69 \%) .{ }^{8}$ Gas in the renal pelvis and ureter is found only in patients with type $2 \mathrm{EPN},{ }^{9}$ in which the prognosis is more favourable, with a mortality rate of $18 \%$.

To our knowledge, 83 Japanese cases of EPN in Japanese subjects have been reported ${ }^{10-12}$ and could be classified into Type 1 or 2, according to Wan's classification. Our case is classified as Type 1, which brings the total number to 84 . These 84 cases include 13 men and 71 women, aged 32 to

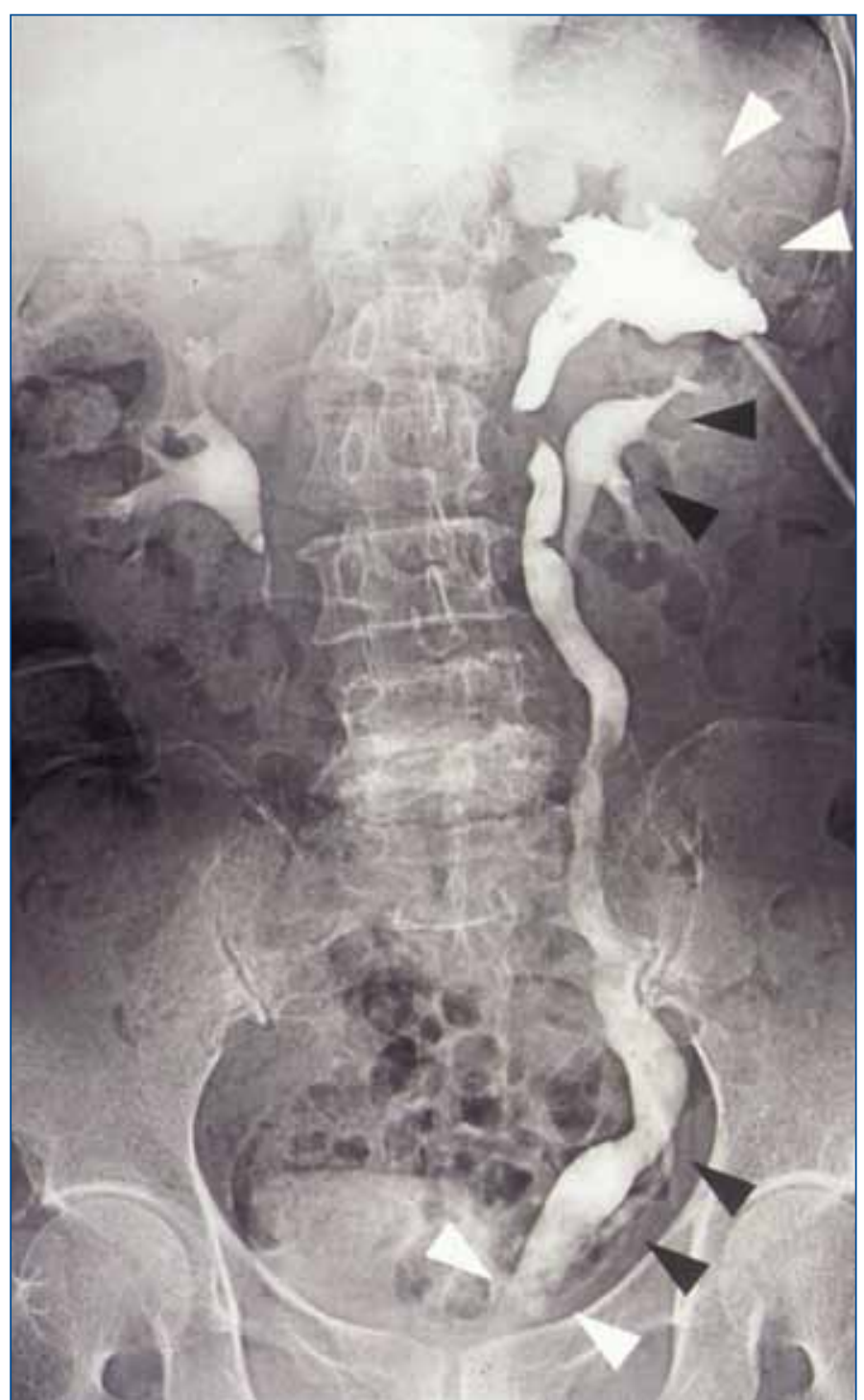

Fig. 2. Intravenous pyelography and antegtrade pyelography show the complete duplication of the left urinary tract. The white arrows show the upper pole moiety and black arrows show the lower pole moiety.

86 years (median 56). Seventeen cases were classified into Type 1 EPN and 67 cases were classified as Type 2. Fiftynine patients $(70.2 \%)$ had diabetes mellitus. There was an average interval of 9.0 days between onset of symptoms and diagnosis. Fifty-two patients (61.9\%) underwent a nephrectomy, 14 patients $(16.7 \%)$ underwent surgical drainage and medical management, and 18 patients $(21.4 \%)$ received medical management alone. The overall mortality rate for EPN patients in this Japanese series was $11.9 \%$ (10/84). Six of the 19 patients $(31.6 \%)$ with Type 1 EPN and 4 of the 61 patients $(6.6 \%)$ with Type 2 I EPN died. There was a significant difference between the mortality rates of patients with Type 1 and Type 2 EPN (chi-square test, $p=0.0026$ ). However, there were no significant differences between 
patients with Type 1 or 2 EPN in terms of background (age, sex and methods of treatment). In addition, the mortality rates in Japanese series were lower than reported by Wan and colleagues. ${ }^{7}$ Wan's classification may be useful to determine treatment for patients with EPN. Patients with Type 1 EPN may need more aggressive treatment methods.

On the other hand, Huang and Tseng also proposed another radiological classification of EPN based on CT: ${ }^{2}$ Class 1: gas in collecting system only; Class 2: parenchymal gas only; Class 3a: extension of gas into perinephric space; Class 3b: extension of gas into pararenal space; and Class 4: EPN in solitary kidney or bilateral disease.

The Class 1 and 2 patients were treated with percutaneous catheter drainage or ureteral catheter combined with antibiotic treatment. Percutaneous catheter drainage should be performed on patients who have localized areas of gas, with functioning kidney tissue. The drainage tubes should stay in place until a follow-up CT scan shows no gas and resolution of inflammation. In the group studied by Huang and Tseng, ${ }^{2}$ Class $3 a$ patients showed a $71 \%$ failure rate following percutaneous drainage, with a $29 \%$ mortality rate; Class 3 b patients showed a $30 \%$ failure rate, with a $19 \%$ mortality rate. The Huang and Tseng system seems appropriate as a management protocol for each disease class. ${ }^{2}$ The 84 Japanese cases of EPN could not be classified according to the Huang and Tseng system due to lack of detailed radiological findings on $\mathrm{CT}$.

According to these criteria, our case is classified as Class $3 \mathrm{a}$ and low-risk. At first, fluids and broad-spectrum antibiotics were administered. Second, a percutaneous nephrostomy was performed through the left kidney. We defined EPN as complete duplication of the left urinary tract and complete obstruction of the upper duplicated tract near the bladder neck. Nevertheless, the condition of the patient relatively improved, and nuclear imaging revealed a nonfunctioning in the left upper pole moiety. According to the flowchart for the management of EPN as per the clinicoradiological classification, ${ }^{2}$ we performed an aggressive nephrectomy. To avoid total nephrectomy and possibility of renal failure, we performed a partial nephrectomy for the affected left upper moiety. Partial nephrectomy for EPN does not appear to be an option for intensive care followed by nephrectomy. However, it seems to be an option in selective cases, such as EPN in a solitary kidney if gas is localized in the upper or lower pole in the affected kidney.

\section{Conclusion}

To the best of our knowledge, this is the first report of EPN in a patient with complete duplication of the left urinary tract. We performed a partial nephrectomy of the affected left upper pole moiety. The patient's postoperative course was satisfactory.

Competing interests: None declared.

This paper has been peer-reviewed.

\section{References}

1. Kelly HA, MacCullum WG. Pneumomaturia. JAMA 1898;31:375-81.

2. Huang JJ, Tseng CC. Emphysematous pyelonephritis. Clinicoradiological classification, management, prognosis and pathogenesis. Arch Intern Med 2000;160:797-805.

3. Pontin AR, Barnes RD. Current management of emphysematous pyelonephritis. Nat Rev Urol 2009;6:272-9.

4. Stein JP, Spitz A, Elmajian DA, et al. Bilateral emphysematous pyelonephritis: a case report and review of the literature. Urology 1996;47:129-34.

5. Shokeir AA, EL-Azab M, Mohsen T, et al. Emphysematous pyelonephritis. A 15 year experience with 20 cases. Urology 1997;49:343-6.

6. Soo Park B, Lee SJ, Wha Kim Y, et al. Outcome of nephrectomy and kidney preserving procedures for the treatment of emphysematous pyelonephritis. Scand I Urol Nephrol 2006;40:332-8.

7. Wan YL, Lee TY, Bullard, et al. Acute gas-producing bacterial renal infection: correlation between imaging findings and clinical outcome. Radiology 1996;198:433-8.

8. Wan YL, Lo SK, Bullard M, et al. Predictors of outcome in emphysematous pyelonephritis. I Urol 1988; 159:369-73

9. Joseph RC, Amendola MA, Artze ME, et al. Genitourinary tract gas: imaging evaluation. Radio Graphics 1996; $16: 295-308$

10. Tsunenori K, Hisashi 0, Mari S, et al. A case of emphysematous pyelonephritis improved with conservative therapy-indication for conservative therapy. Acta Urol Jpn 2000;46:335-8.

11. Yukihiro U, Takaichiro I, Masayuki T, et al. Emphysematous pyelonephritis complicated with diabetes mellitus: A case report. Acta Urol Jpn 1999;45:477-80.

12. Yoshikazu F, Shin-ichi T, Shin-ichi K, et al. Emphysematous pyelonephritis complicated with diabetes mellitus: A case report. Nishinihon J Urol 1991;53:1470-4.

Correspondence: Dr. Yasuto Yamasaki, Department of Urology, Deputy Director, Japanese Red Cross Nagasaki Genbaku Hospital, 3-15 Mori-machi , Nagasaki city, Nagasaki, 852-851 1 Japan; fax: +81 (95) 847-8036; anjin07@nagasaki-med.jrc.or.jp 